\begin{tabular}{|c|l|}
\hline Title & Pseudo-spherical evolutes of curves on a spacelike surface in three dimensional Lorentz-Minkowski space \\
\hline Author(s) & Sato, Takami \\
\hline Citation & Hokkaido University Preprint Series in Mathematics, 1000, 1-12 \\
\hline Issue Date & 2012-39 \\
\hline DOI & 10.14943/84146 \\
\hline Doc URL & http://hdl.handle.net/2115/69805 \\
\hline Type & bulletin (article) \\
\hline File Information & pre1000.pdf \\
\hline
\end{tabular}

Instructions for use 


\title{
Pseudo-spherical evolutes of curves on a spacelike surface in three dimensional Lorentz-Minkowski space
}

\author{
Takami SATO
}

March 9, 2012

\begin{abstract}
In this paper we introduce the notion of pseudo-spherical evolutes of curves on a spacelike surface in three dimensional Lorentz-Minkowski space which is analogous to the notion of evolutes of curves on the hyperbolic plane. We investigate the the singularities and geometric properties of pseudo-spherical evolutes of curves on a spacelike surface.
\end{abstract}

\section{Introduction}

In this paper we study the the singularities of pseudo-spherical evolutes of curves on a spacelike surface in three dimensional Lorentz-Minkowski space. The study of the extrinsic differential geometry of submanifolds in Minkowski space is of special interest in relativity theory. There are some papers that investigate codimension two spacelike submanifolds in Lorentz-Minkowski space, for instance, in [3]. Inspired by these papers, we are particularly interested in spacelike curves in three dimensional Lorentz-Minkowski space as a special case, that is, submanifolds of codimension two in the space. As an application of the idea in [3], we considered curves on a spacelike surface in three dimensional Lorentz-Minkowski space in the last paper [6]. Moreover in [2], it was investigated the evolutes of hyperbolic plane curves and those geometric properties. Inspired by the above results, we introduce the notion of pseudo-spherical evolutes of curves on a spacelike surface in three dimensional Lorentz-Minkowski space and investigate the geometric propoerties. The principal tools for the study of evolutes of hyperbolic plane are the hyperbolic Frenet-Serret formula and hyperbolic height functions on hyperbolic plane curves (cf., [2]). In our case, we adopt a special pseudo-orthonormal frame in $\mathbb{R}_{1}^{3}$ which is called the Lorentzian Darboux frame and have the Frenet-Serret type formulae (cf., $\S 2$ ). Since we consider a spacelike surface $M$, we can choose a future directed unit timelike normal vector field $\boldsymbol{n}$ along the surface. For a curve $\boldsymbol{\gamma}$ on the surface, we restrict the normal vector field $\boldsymbol{n}$ along $\boldsymbol{\gamma}$, so that we have a unit timelike normal vector field $\boldsymbol{n}_{\boldsymbol{\gamma}}$ along $\boldsymbol{\gamma}$. Moreover, we choose the unit tangent vector field $\boldsymbol{t}$ and another normal vector field $\boldsymbol{b}$ along $\gamma$. As a result, we construct a pseudo-orthonormal frame $\left\{\boldsymbol{t}, \boldsymbol{n}_{\boldsymbol{\gamma}}, \boldsymbol{b}\right\}$ along the curve $\boldsymbol{\gamma}$. We define two families of functions on a curve, timelike height function $H^{T}$ and spacelike height function $H^{S}$. By differentiating these functions, we

2010 Mathematics Subject classification: 53B30, 53A35

Key Words and Phrases: Curves on a spacelike surface, Pseudo-spherical evolute, Lorentz-Minkowski space 
obtain new invariants $\sigma_{H}$ and $\sigma_{D}$ which characterize the each conditions of derivation of $H^{T}$ and $H^{S}$. For instance, $\frac{\partial H^{T}}{\partial t}=\frac{\partial^{2} H^{T}}{\partial t^{2}}=\frac{\partial^{3} H^{T}}{\partial t^{3}}=0$ if and only if $\sigma_{H}=0$ and some other conditions. In $\S 3$, we also define two important curves $\boldsymbol{h}_{\boldsymbol{\gamma}}$ in the hyperbolic space and $\boldsymbol{d}_{\boldsymbol{\gamma}}$ in de Sitter space by observing the conditions of first and second derivation of $H^{T}$ and $H^{S}$ respectively. We call $\boldsymbol{h}_{\boldsymbol{\gamma}}$ a hyperbolic evolute of $\boldsymbol{\gamma}$ relative to $M$ and $\boldsymbol{d}_{\boldsymbol{\gamma}}$ a de Sitter evolute of $\boldsymbol{\gamma}$ relative to $M$. We show that the hyperbolic evolute $\boldsymbol{h}_{\boldsymbol{\gamma}}$ is constant if and only if $\sigma_{H} \equiv 0$. In this case the curve $\boldsymbol{\gamma}$ is a special curve on the surface $M$, which is called a hyperbolic-slice (or an $H$-slice) of $M$. We also show that the de Sitter evolute $\boldsymbol{d}_{\boldsymbol{\gamma}}$ is constant if and only if $\sigma_{D} \equiv 0$ and define a special curve on the surface $M$ called a de Sitter-slice(or an D-slice) of $M$. We consider H-slice and D-slice of $M$ as the model curves on the surface $M$. Moreover where $\sigma_{H}=0$ and $\sigma_{H}^{\prime} \neq 0$, it is a point where $\gamma$ has third order contact with one of the tangent $\mathrm{H}$-slices of $M$, and we also the same argument about $\gamma$ and tangent D-slice where $\sigma_{D}=0$ and $\sigma_{D}^{\prime} \neq 0$. Here we remark that tangent D-slice is always non-singular, but we have the case that H-slice has a singular point (cf., §3). As an application of the theory of unfoldings of functions in [1], we give a classification of singularities of both the hyperbolic evolute and the de Sitter evolute in Theorem 5.4, which is one of the main results in this paper.

We explain in $\S 2$ the basic notions of Lorentz-Minkowski space and introduce Lorentzian Darboux frame that will be used throughout the paper. In $\S 3$ is devoted to the study of two important height functions, the hyperbolic height function and the de Sitter height function by considering the relationship with invariants $\sigma_{H}$ and $\sigma_{D}$. Moreover, in $\S 4$, we have the main results in this paper that local properties of the curve and the hyperbolic evolute and the de Sitter evolute of the curve provided by the invariant $\sigma_{H}$ and $\sigma_{D}$. Finally in $\S 5$ we consider Euclidean plane curves and the hyperbolic plane curves and curves on a surface of the graph of a function as a special case.

\section{Definitions and Basic Facts}

In this section we prepare some definitions and basic facts which we will use in this paper. For basic concepts and details of properties, see $[5,6]$. Let $\mathbb{R}^{3}$ be a three-dimensional vector space. For any $\boldsymbol{x}=\left(x_{0}, x_{1}, x_{2}\right), \boldsymbol{y}=\left(y_{0}, y_{1}, y_{2}\right) \in \mathbb{R}^{3}$, the pseudo-scalar product of $\boldsymbol{x}$ and $\boldsymbol{y}$ is defined by $\langle\boldsymbol{x}, \boldsymbol{y}\rangle=-x_{0} y_{0}+x_{1} y_{1}+x_{2} y_{2}$. We call $\left(\mathbb{R}^{3},\langle\rangle,\right)$ Minkowski 3 -space. We write $\mathbb{R}_{1}^{3}$ instead of $\left(\mathbb{R}^{3},\langle\rangle,\right)$. We say that a non-zero vector $\boldsymbol{x} \in \mathbb{R}_{1}^{3}$ is spacelike, lightlike or timelike if $\langle\boldsymbol{x}, \boldsymbol{x}\rangle>0,\langle\boldsymbol{x}, \boldsymbol{x}\rangle=0$ or $\langle\boldsymbol{x}, \boldsymbol{x}\rangle<0$ respectively. The norm of the vector $\boldsymbol{x} \in \mathbb{R}_{1}^{3}$ is defined by $\|\boldsymbol{x}\|=\sqrt{|\langle\boldsymbol{x}, \boldsymbol{x}\rangle|}$. Here we define the notion of planes. For a non-zero vector $\boldsymbol{v} \in \mathbb{R}_{1}^{3}$ and a real number c, we define a plane with pseudo-normal $\boldsymbol{v}$ by

$$
P(\boldsymbol{v}, c)=\left\{\boldsymbol{x} \in \mathbb{R}_{1}^{3} \mid\langle\boldsymbol{x}, \boldsymbol{v}\rangle=c\right\} .
$$

We call $P(\boldsymbol{v}, c)$ a spacelike plane, a timelike plane or a lightlike plane if $\boldsymbol{v}$ is timelike, spacelike or lightlike respectively. We now define Hyperbolic plane by

$$
H_{+}^{2}(-1)=\left\{\boldsymbol{x} \in \mathbb{R}_{1}^{3} \mid\langle\boldsymbol{x}, \boldsymbol{x}\rangle=-1, x_{0}>0\right\}
$$

and de Sitter 2-space by

$$
S_{1}^{2}=\left\{\boldsymbol{x} \in \mathbb{R}_{1}^{3} \mid\langle\boldsymbol{x}, \boldsymbol{x}\rangle=1\right\}
$$

Here we define

$$
\boldsymbol{a} \wedge \boldsymbol{b}=\left|\begin{array}{ccc}
-\boldsymbol{e}_{0} & \boldsymbol{e}_{1} & \boldsymbol{e}_{2} \\
a_{0} & a_{1} & a_{2} \\
b_{0} & b_{1} & b_{2}
\end{array}\right|
$$


where $\boldsymbol{a}=\left(a_{0}, a_{1}, a_{2}\right), \boldsymbol{b}=\left(b_{0}, b_{1}, b_{2}\right)$ and $\left\{\boldsymbol{e}_{0}, \boldsymbol{e}_{1}, \boldsymbol{e}_{2}\right\}$ is the canonical basis of $\mathbb{R}^{3}$.

We now prepare some basic facts of curves on a spacelike surface. We consider a spacelilke embedding $\boldsymbol{X}: U \longrightarrow \mathbb{R}_{1}^{3}$ from an open subset $U \subset \mathbb{R}^{2}$. We write $M=\boldsymbol{X}(U)$ and identify $M$ and $U$ through the embedding $\boldsymbol{X}$. Here, we say that $\boldsymbol{X}$ is a spacelike embedding if the tangent space $T_{p} M$ consists of spacelike vectors at any $p=\boldsymbol{X}(u)$. Let $\bar{\gamma}: I \longrightarrow U$ be a regular curve and we have a curve $\boldsymbol{\gamma}: I \longrightarrow M \subset \mathbb{R}_{1}^{3}$ defined by $\boldsymbol{\gamma}(s)=\boldsymbol{X}(\overline{\boldsymbol{\gamma}}(s))$. We say that $\boldsymbol{\gamma}$ is a curve on the spacelike surface $M$. Since $\boldsymbol{\gamma}$ is a spacelike curve, we can reparameterize it by the arc-length $s$. So we have the unit tangent vector $\boldsymbol{t}(s)=\boldsymbol{\gamma}^{\prime}(s)$ of $\boldsymbol{\gamma}(s)$. Since $\boldsymbol{X}$ is a spacelike embedding, we have a unit timelike normal vector field $\boldsymbol{n}$ along $M=\boldsymbol{X}(U)$ defined by

$$
\boldsymbol{n}(p)=\frac{\boldsymbol{X}_{u_{1}}(u) \wedge \boldsymbol{X}_{u_{2}}(u)}{\left\|\boldsymbol{X}_{u_{1}}(u) \wedge \boldsymbol{X}_{u_{2}}(u)\right\|}
$$

for $p=\boldsymbol{X}(u)$. We say that $\boldsymbol{n}$ is future directed if $\left\langle\boldsymbol{n}, \boldsymbol{e}_{0}\right\rangle<0$. We choose the orientation of $M$ such that $\boldsymbol{n}$ is future directed. We define $\boldsymbol{n}_{\boldsymbol{\gamma}}(s)=\boldsymbol{n} \circ \gamma(s)$, so that we have a unit timelike normal vector field $\boldsymbol{n}_{\boldsymbol{\gamma}}$ along $\boldsymbol{\gamma}$. Therefore we can construct a spacelike unit normal section $\boldsymbol{b}(s) \in N_{p}(M)$ by $\boldsymbol{b}(s)=\boldsymbol{t}(s) \wedge \boldsymbol{n}_{\gamma}(s)$. It follows that we have $\left\langle\boldsymbol{n}_{\gamma}, \boldsymbol{n}_{\gamma}\right\rangle=-1,\left\langle\boldsymbol{n}_{\gamma}, \boldsymbol{b}\right\rangle=$ $0,\langle\boldsymbol{b}, \boldsymbol{b}\rangle=1$. Then we have a pseudo-orthonormal frame $\left\{\boldsymbol{t}(s), \boldsymbol{n}_{\gamma}(s), \boldsymbol{b}(s)\right\}$, which is called the Lorentzian Darboux frame along $\gamma$. By standard arguments, we have the following Frenet-Serret type formulae:

$$
\left\{\begin{array}{l}
\boldsymbol{t}^{\prime}(s)=\kappa_{n}(s) \boldsymbol{n}_{\gamma}(s)+\kappa_{g}(s) \boldsymbol{b}(s) \\
\boldsymbol{n}_{\gamma}^{\prime}(s)=\kappa_{n}(s) \boldsymbol{t}(s)+\tau_{g}(s) \boldsymbol{b}(s) \\
\boldsymbol{b}^{\prime}(s)=-\kappa_{g}(s) \boldsymbol{t}(s)+\tau_{g}(s) \boldsymbol{n}_{\gamma}(s),
\end{array}\right.
$$

where $\kappa_{n}(s)=-\left\langle\boldsymbol{t}^{\prime}(s), \boldsymbol{n}_{\gamma}(s)\right\rangle, \kappa_{g}(s)=\left\langle\boldsymbol{t}^{\prime}(s), \boldsymbol{b}(s)\right\rangle$ and $\tau_{g}(s)=-\left\langle\boldsymbol{b}^{\prime}(s), \boldsymbol{n}_{\gamma}(s)\right\rangle$. Here, we have the following properties of $\gamma$ characterized by the conditions of $\kappa_{g}, \kappa_{n}, \tau_{g}$.

$$
\gamma \text { is }\left\{\begin{array}{l}
\text { a geodesic curve if and only if } \kappa_{g} \equiv 0 \\
\text { an acymptotic curve if and only if } \kappa_{n} \equiv 0 \\
\text { a principal curve if and only if } \tau_{g} \equiv 0
\end{array}\right.
$$

\section{$3 \quad$ Height Functions}

In this section, we introduce two families of functions on a curve on a spacelike surface $\mathrm{M}$ $\gamma: I \longrightarrow M \subset \mathbb{R}_{1}^{3}$. We now define two families of functions as follow:

$$
\begin{gathered}
H^{T}: I \times H_{+}^{2}(-1) \longrightarrow \mathbb{R} ; \quad(s, \boldsymbol{v}) \longmapsto\langle\gamma(s), \boldsymbol{v}\rangle, \\
H^{S}: I \times S_{1}^{2} \longrightarrow \mathbb{R} ; \quad(s, \boldsymbol{v}) \longmapsto\langle\gamma(s), \boldsymbol{v}\rangle,
\end{gathered}
$$

We call $H^{T}$ the timelike height function of $\gamma$ on $\mathrm{M}$ and $H^{S}$ the spacelike height function of $\boldsymbol{\gamma}$ on M. We denote $h_{\boldsymbol{v}}^{T}(s)=H^{T}(s, \boldsymbol{v})$ for any fixed $\boldsymbol{v} \in H_{+}^{2}(-1)$ and $h_{\boldsymbol{v}}^{S}(s)=H^{S}(s, \boldsymbol{v})$ for any fixed $\boldsymbol{v} \in S_{1}^{2}$.

Here we calculate the derivative of $H^{T}$. By Frenet-Serret type formulae, we have

$$
\frac{\partial H^{T}}{\partial s}=\left\langle\gamma^{\prime}(s), \boldsymbol{v}\right\rangle=\langle\boldsymbol{t}, \boldsymbol{v}\rangle=0
$$


Since $\boldsymbol{v} \in H_{+}^{2}(-1)$, there are $\lambda, \mu \in \mathbb{R}$ such that $\boldsymbol{v}=\lambda \boldsymbol{n}_{\gamma}(s)+\mu \boldsymbol{b}(s)$ and $-\lambda^{2}+\mu^{2}=-1$ if $\frac{\partial H^{T}}{\partial s}=0$. Here we have

$$
\frac{\partial^{2} H^{T}}{\partial s^{2}}=\left\langle\boldsymbol{t}^{\prime}(s), \boldsymbol{v}\right\rangle=\left\langle\kappa_{n} \boldsymbol{n}_{\boldsymbol{\gamma}}+\kappa_{g} \boldsymbol{b}, \lambda \boldsymbol{n}_{\boldsymbol{\gamma}}+\mu \boldsymbol{b}\right\rangle=-\lambda \kappa_{n}+\mu \kappa_{g}=0 .
$$

Therefore, $\frac{\partial H^{T}}{\partial s}=\frac{\partial^{2} H^{T}}{\partial s^{2}}=0$ if and only if $\boldsymbol{v}=\lambda \boldsymbol{n}_{\gamma}(s)+\mu \boldsymbol{b}(s),-\lambda^{2}+\mu^{2}=-1$ and $\lambda \kappa_{n}=\mu \kappa_{g}$. It means that

$$
\lambda^{2}\left(\kappa_{g}^{2}-\kappa_{n}^{2}\right)=\kappa_{g}^{2}
$$

Since $\lambda, \mu \in \mathbb{R}$, we can consider under the condition $\kappa_{g}^{2}>\kappa_{n}^{2}$, then we have

$$
\boldsymbol{v}= \pm \frac{1}{\sqrt{\kappa_{g}^{2}(s)-\kappa_{n}^{2}(s)}}\left(\kappa_{g}(s) \boldsymbol{n}_{\boldsymbol{\gamma}}(s)+\kappa_{n}(s) \boldsymbol{b}(s)\right)
$$

Moreover we have that $\frac{\partial H^{T}}{\partial s}=\frac{\partial^{2} H^{T}}{\partial s^{2}}=\frac{\partial^{3} H^{T}}{\partial s^{3}}=0$ if and only if

$$
\kappa_{g} \kappa_{n}{ }^{\prime}+\kappa_{g}^{2} \tau_{g}-\kappa_{n}^{2} \tau_{g}-\kappa_{n} \kappa_{g}{ }^{\prime}=0 .
$$

Under the condition $\kappa_{g}^{2}>\kappa_{n}^{2}$, (which implies $\kappa_{g}^{2}>0$,) the equation $\left(^{*}\right)$ is divided by $\kappa_{g}^{2}$, so that we have

$$
\left(\frac{\kappa_{n}}{\kappa_{g}}\right)^{\prime}+\tau_{g}\left(1-\left(\frac{\kappa_{n}}{\kappa_{g}}\right)^{2}\right)=0 .
$$

We now define $\sigma_{H}$ by $\sigma_{H}=\left(\frac{\kappa_{n}}{\kappa_{g}}\right)^{\prime}+\tau_{g}\left(1-\left(\frac{\kappa_{n}}{\kappa_{g}}\right)^{2}\right)$. If we calculate the 4 th derivative of $H^{S}$, we can show that the above conditions with the extra condition

$$
\frac{\partial^{4} H^{T}}{\partial s^{4}}=-\kappa_{g} \kappa_{n}{ }^{\prime \prime}-2 \kappa_{g} \kappa_{g}{ }^{\prime} \tau_{h}-\kappa_{g}{ }^{2} \tau_{g}{ }^{\prime}+2 \kappa_{n} \kappa_{n}{ }^{\prime} \tau_{g}+\kappa_{n}{ }^{2} \tau_{g}{ }^{2}+\kappa_{n} \kappa_{g}{ }^{\prime \prime}=0
$$

is equivalent to the conditions $\sigma_{H}(s)=0$ and $\left(\sigma_{H}\right)^{\prime}(s)=0$.

Moreover, we calculate the derivatives of $H^{S}$ under the condition $\kappa_{n}^{2}>\kappa_{g}^{2}$, (which implies $\kappa_{n}^{2}>0$, ) as the above, and we also define $\sigma_{D}=\left(\frac{\kappa_{g}}{\kappa_{n}}\right)^{\prime}+\tau_{g}\left(1-\left(\frac{\kappa_{g}}{\kappa_{n}}\right)^{2}\right)$.

As a consequence, we have the following proposition:

Proposition 3.1 (A) For any $(s, \boldsymbol{v}) \in I \times H^{2}(-1)$, we have the followings:

(1) $\left(h_{\boldsymbol{v}}^{T}\right)^{\prime}(s)=0$ if and only if $\boldsymbol{v}=\lambda \boldsymbol{n}_{\gamma}(s)+\mu \boldsymbol{b}(s)$ where $\lambda, \mu \in \mathbb{R}$ such that $-\lambda^{2}+\mu^{2}=-1$.

(2) $\left(h_{\boldsymbol{v}}^{T}\right)^{\prime}(s)=\left(h_{\boldsymbol{v}}^{T}\right)^{\prime \prime}(s)=0$ if and only if

$$
\boldsymbol{v}= \pm \frac{1}{\sqrt{\kappa_{g}^{2}(s)-\kappa_{n}^{2}(s)}}\left(\kappa_{g}(s) \boldsymbol{n}_{\boldsymbol{\gamma}}(s)+\kappa_{n}(s) \boldsymbol{b}(s)\right)
$$

and $\kappa_{g}^{2}(s)>\kappa_{n}^{2}(s)$.

(3) $\left(h_{\boldsymbol{v}}^{T}\right)^{\prime}(s)=\left(h_{\boldsymbol{v}}^{T}\right)^{\prime \prime}(s)=\left(h_{\boldsymbol{v}}^{T}\right)^{\prime \prime \prime}(s)=0$ if and only if

$$
\boldsymbol{v}= \pm \frac{1}{\sqrt{\kappa_{g}^{2}(s)-\kappa_{n}^{2}(s)}}\left(\kappa_{g}(s) \boldsymbol{n}_{\boldsymbol{\gamma}}(s)+\kappa_{n}(s) \boldsymbol{b}(s)\right)
$$


$\kappa_{g}^{2}(s)>\kappa_{n}^{2}(s)$, and $\sigma_{H}=0$.

(4) $\left(h_{\boldsymbol{v}}^{T}\right)^{\prime}(s)=\left(h_{\boldsymbol{v}}^{T}\right)^{\prime \prime}(s)=\left(h_{\boldsymbol{v}}^{T}\right)^{\prime \prime \prime}(s)=\left(h_{\boldsymbol{v}}^{T}\right)^{(4)}(s)=0$ if and only if

$$
\boldsymbol{v}= \pm \frac{1}{\sqrt{\kappa_{g}^{2}(s)-\kappa_{n}^{2}(s)}}\left(\kappa_{g}(s) \boldsymbol{n}_{\boldsymbol{\gamma}}(s)+\kappa_{n}(s) \boldsymbol{b}(s)\right)
$$

$\kappa_{g}^{2}(s)>\kappa_{n}^{2}(s), \sigma_{H}=0$ and $\left(\sigma_{H}\right)^{\prime}=0$.

(B) For any $(s, \boldsymbol{v}) \in I \times S_{1}^{2}$, we have the followings:

(1) $\left(h_{\boldsymbol{v}}^{S}\right)^{\prime}(s)=0$ if and only if $\boldsymbol{v}=\lambda \boldsymbol{n}_{\gamma}(s)+\mu \boldsymbol{b}(s)$ where $\lambda, \mu \in \mathbb{R}$ such that $-\lambda^{2}+\mu^{2}=-1$.

(2) $\left(h_{\boldsymbol{v}}^{S}\right)^{\prime}(s)=\left(h_{\boldsymbol{v}}^{S}\right)^{\prime \prime}(s)=0$ if and only if

$$
\boldsymbol{v}= \pm \frac{1}{\sqrt{\kappa_{n}^{2}(s)-\kappa_{g}^{2}(s)}}\left(\kappa_{g}(s) \boldsymbol{n}_{\boldsymbol{\gamma}}(s)-\kappa_{n}(s) \boldsymbol{b}(s)\right)
$$

and $\kappa_{n}^{2}(s)>\kappa_{g}^{2}(s)$.

(3) $\left(h_{\boldsymbol{v}}^{S}\right)^{\prime}(s)=\left(h_{\boldsymbol{v}}^{S}\right)^{\prime \prime}(s)=\left(h_{\boldsymbol{v}}^{S}\right)^{\prime \prime \prime}(s)=0$ if and only if

$$
\boldsymbol{v}= \pm \frac{1}{\sqrt{\kappa_{n}^{2}(s)-\kappa_{g}^{2}(s)}}\left(\kappa_{g}(s) \boldsymbol{n}_{\boldsymbol{\gamma}}(s)-\kappa_{n}(s) \boldsymbol{b}(s)\right),
$$

$\kappa_{n}^{2}(s)>\kappa_{g}^{2}(s)$, and $\sigma_{D}=0$.

(4) $\left(h_{\boldsymbol{v}}^{S}\right)^{\prime}(s)=\left(h_{\boldsymbol{v}}^{S}\right)^{\prime \prime}(s)=\left(h_{\boldsymbol{v}}^{S}\right)^{\prime \prime \prime}(s)=\left(h_{\boldsymbol{v}}^{S}\right)^{(4)}(s)=0$ if and only if

$$
\boldsymbol{v}= \pm \frac{1}{\sqrt{\kappa_{n}^{2}(s)-\kappa_{g}^{2}(s)}}\left(\kappa_{g}(s) \boldsymbol{n}_{\boldsymbol{\gamma}}(s)-\kappa_{n}(s) \boldsymbol{b}(s)\right)
$$

$\kappa_{n}^{2}(s)>\kappa_{g}^{2}(s), \sigma_{D}=0$ and $\left(\sigma_{D}\right)^{\prime}=0$.

By the above proposition, we have two invariants $\sigma_{H}$ and $\sigma_{D}$.

We now respectively define curves $\boldsymbol{h}_{\boldsymbol{\gamma}}: I \longrightarrow H_{+}^{2}(-1)$ and $\boldsymbol{d}_{\boldsymbol{\gamma}}: I \longrightarrow S_{1}^{2}$ by

$$
\begin{aligned}
& \boldsymbol{h}_{\boldsymbol{\gamma}}(s)=\frac{\kappa_{g}(s)}{\sqrt{\kappa_{g}^{2}(s)-\kappa_{n}^{2}(s)}} \boldsymbol{n}_{\boldsymbol{\gamma}}(s)+\frac{\kappa_{n}(s)}{\sqrt{\kappa_{g}^{2}(s)-\kappa_{n}^{2}(s)}} \boldsymbol{b}(s) \\
& \boldsymbol{d}_{\boldsymbol{\gamma}}(s)=\frac{\kappa_{g}(s)}{\sqrt{\kappa_{n}^{2}(s)-\kappa_{g}^{2}(s)}} \boldsymbol{n}_{\boldsymbol{\gamma}}(s)-\frac{\kappa_{n}(s)}{\sqrt{\kappa_{n}^{2}(s)-\kappa_{g}^{2}(s)}} \boldsymbol{b}(s)
\end{aligned}
$$

We call $\boldsymbol{h}_{\boldsymbol{\gamma}}$ a hyperbolic evolute of $\gamma$ relative to $M$ and $\boldsymbol{d}_{\boldsymbol{\gamma}}$ a de Sitter evolute of $\boldsymbol{\gamma}$ relative to $M$ respectively. We also call one of $\boldsymbol{h}_{\boldsymbol{\gamma}}$ and $\boldsymbol{d}_{\boldsymbol{\gamma}}$ a pseudo-spherical evolute of $\boldsymbol{\gamma}$ relative to M. By straightforward calculations, we have $\boldsymbol{h}_{\boldsymbol{\gamma}}^{\prime}(s)=0$ if and only if $\sigma_{H}(s)=0$. Moreover, we have $\boldsymbol{d}_{\boldsymbol{\gamma}}^{\prime}(s)=0$ if and only if $\sigma_{D}(s)=0$. Therefore, $\boldsymbol{h}_{\boldsymbol{\gamma}}(s)=\boldsymbol{v}_{0}$ is constant if and only if $\sigma_{H}(s) \equiv 0$. In this case, by Proposition $3.1, h_{\boldsymbol{v}_{0}}^{T}(s)$ is constant, that is, there is a real number $c \in \mathbb{R}$ such that $\left\langle\gamma(s), \boldsymbol{v}_{0}\right\rangle=c$. It means that $\operatorname{Im} \boldsymbol{\gamma}=P\left(\boldsymbol{v}_{0}, c\right) \cap M$. It suggests that curves of the form $P(\boldsymbol{v}, c) \cap M$ for $\boldsymbol{v} \in H_{+}^{2}(-1)$ are the candidates of model curves on $M$. These might play a similar role to lines in Euclidean plane. We call it a hyperbolic-slice (or, an H-slice) of M. By the same way, we can also define de Sitter-slice (or, a $D$-slice) of $M$ by $P(\boldsymbol{v}, c) \cap M$ for $\boldsymbol{v} \in S_{1}^{2}$. 
Here we remark that we can consider the above H-slice under the condition $\kappa_{n} \neq 0$. If $\kappa_{n}=0$, we have $\boldsymbol{h}_{\boldsymbol{\gamma}}(s)=\boldsymbol{n}_{\boldsymbol{\gamma}}$. It means that $P(\boldsymbol{v}, c)$ is tangent space at $p=\boldsymbol{X}(u)$ for $\boldsymbol{v} \in H_{+}^{2}(-1)$ and $P(\boldsymbol{v}, c) \cap M$ has a singular point.

We consider the geometric meanings of the invariants $\sigma_{H}$ and $\sigma_{D}$. For the purpose, we introduce the following another family of function:

$$
\mathcal{H}^{T}: \mathbb{R}_{1}^{3} \times H_{+}^{2}(-1) \longrightarrow \mathbb{R} ; \quad(\boldsymbol{x}, \boldsymbol{v}) \longmapsto\langle\boldsymbol{x}, \boldsymbol{v}\rangle .
$$

We denote $\mathfrak{h}_{\boldsymbol{v}_{0}}^{T}(\boldsymbol{x})=\mathcal{H}^{T}\left(\boldsymbol{x}, \boldsymbol{v}_{0}\right)$ for any fixed $\boldsymbol{v}_{0} \in H_{+}^{2}(-1)$, then, we have

$$
h_{\boldsymbol{v}_{0}}^{T}(s)=\left\langle\gamma(s), \boldsymbol{v}_{0}\right\rangle=\mathcal{H}\left(\boldsymbol{\gamma}(s), \boldsymbol{v}_{0}\right)=\mathfrak{h}_{\boldsymbol{v}_{0}}^{T}(\boldsymbol{\gamma}(s))
$$

Moreover, for any $s_{0} \in \mathbb{R}$ and $\boldsymbol{v}_{0}=\boldsymbol{h}_{\boldsymbol{\gamma}}\left(s_{0}\right),\left(\left.\mathfrak{h}_{\boldsymbol{v}_{0}}^{T}\right|_{M}\right)^{-1}(c)$ is an H-slice of $M$. By Proposition $3.1,\left(\mathfrak{h}_{\boldsymbol{v}_{0}}^{T}\right)^{-1}\left(c_{0}\right)=P\left(\boldsymbol{v}_{0}, c_{0}\right)$ is tangent to $\gamma$ at $\boldsymbol{\gamma}\left(s_{0}\right)$, so that $\left(\left.\mathfrak{h}_{\boldsymbol{v}_{0}}^{T}\right|_{M}\right)^{-1}\left(c_{0}\right)$ is an H-slice of $M$ tangent to $\boldsymbol{\gamma}$ at $\boldsymbol{\gamma}\left(s_{0}\right)$, where $c_{0}=h_{\boldsymbol{v}_{0}}^{T}\left(s_{0}\right)$. We denote that $T P_{\boldsymbol{v}_{0}, \boldsymbol{\gamma}\left(s_{0}\right)}^{S}=P\left(\boldsymbol{v}_{0}, c_{0}\right)$ which is called a spacelike tangent plane of $\boldsymbol{\gamma}$ at $\boldsymbol{\gamma}\left(s_{0}\right)$ with respect to $\boldsymbol{v}_{0}=\boldsymbol{h}_{\boldsymbol{\gamma}}\left(s_{0}\right)$. Moreover, if $\kappa_{n}\left(s_{0}\right) \neq 0$, then the H-slice $\left(\left.\mathfrak{h}_{\boldsymbol{v}_{0}}^{T}\right|_{M}\right)^{-1}\left(c_{0}\right)$ is non-singular at $\boldsymbol{\gamma}\left(s_{0}\right)$. Then we call it a tangent H-slice of $\boldsymbol{\gamma}$ at $\boldsymbol{\gamma}\left(s_{0}\right)$ relative to $M$. It is denoted by $T_{M, \gamma\left(s_{0}\right)}^{H}$. Now let $F: \mathbb{R}_{1}^{3} \longrightarrow \mathbb{R}$ (respectively, $\left.F\right|_{M}: M \longrightarrow \mathbb{R}$ ) be a submersion and $\gamma\left(s_{0}\right) \subset F^{-1}(0) \cap M$. We say that $\gamma$ and $F^{-1}(0)$ (respectively, $\left.F^{-1}(0) \cap M\right)$ have contact of order $k$ if the function $f=F \circ \gamma(s)$ satisfies $f\left(s_{0}\right)=f^{\prime}\left(s_{0}\right)=\cdots=f^{(k)}\left(s_{0}\right)=0$ and $f^{(k+1)}\left(s_{0}\right) \neq 0$. Under the above notations and by Proposition 3.1, we can conclude $\gamma$ and $T P_{\boldsymbol{v}_{0}, \gamma\left(s_{0}\right)}^{S}$ have contact of order three at $\gamma\left(s_{0}\right)$ if and only if $\sigma_{H}\left(s_{0}\right)=0$ and $\sigma_{H}^{\prime}\left(s_{0}\right) \neq 0$. Under the assumption that $\kappa_{n}\left(s_{0}\right) \neq 0$ the above conditions are equivalent to the condition that $\gamma$ and $T_{M, \gamma\left(s_{0}\right)}^{H}$ have contact of order three at $\gamma\left(s_{0}\right)$. Therefore, we have the following proposition:

Proposition 3.2 Let $\gamma: I \longrightarrow M$ be a regular curve on $M$. Then the following conditions are equivalent:

(1) $\boldsymbol{\gamma}$ and the spacelike tangent plane $T P_{\boldsymbol{v}_{0}, \boldsymbol{\gamma}\left(s_{0}\right)}^{S}$ have contact order three,

(2) $\sigma_{H}\left(s_{0}\right)=0$ and $\sigma_{H}^{\prime}\left(s_{0}\right) \neq 0$.

If $\kappa_{n}\left(s_{0}\right) \neq 0$, then the tangent $H$-slice $T_{M, \gamma\left(s_{0}\right)}^{H}$ of $\boldsymbol{\gamma}$ at $\gamma\left(s_{0}\right)$ is non-singular and the above two conditions are equivalent to the following condition:

(3) $\gamma$ and the the tangent $H$-slice $T_{M, \gamma\left(s_{0}\right)}^{H}$ have contact order three.

Moreover, if $\kappa_{n}\left(s_{0}\right)=0$ then the spacelike tangent plane $T P_{\boldsymbol{v}_{0}, \boldsymbol{\gamma}\left(s_{0}\right)}^{S}$ is the tangent plane $T M_{\gamma\left(s_{0}\right)}$ of $M$ and $\boldsymbol{t}\left(s_{0}\right)$ is an asymptotic direction of $M$ at $\gamma\left(s_{0}\right)$.

On the other hand, $P(\boldsymbol{v}, c)$ for $\boldsymbol{v} \in S_{1}^{2}$ is a timelike plane. Therefore a D-slice is always a regular curve. We also define

$$
\mathcal{H}^{S}: \mathbb{R}_{1}^{3} \times S_{1}^{2} \longrightarrow \mathbb{R} ; \quad(\boldsymbol{x}, \boldsymbol{v}) \longmapsto\langle\boldsymbol{x}, \boldsymbol{v}\rangle .
$$

By exactly the same arguments as the above case, we have the notions of timelike tangent plane of $\boldsymbol{\gamma}$ at $\boldsymbol{\gamma}\left(s_{0}\right)$ with respect to $\boldsymbol{v}_{0}=\boldsymbol{d}_{\boldsymbol{\gamma}}\left(s_{0}\right)$ and tangent D-slice of $\boldsymbol{\gamma}$ at $\boldsymbol{\gamma}\left(s_{0}\right)$ relative to $M$, respectively. We respectively denote these as $T P_{\boldsymbol{v}_{0}, \boldsymbol{\gamma}\left(s_{0}\right)}^{T}$ and $T_{M, \boldsymbol{\gamma}\left(s_{0}\right)}^{D}$. In this case the tangent $D$-slice is always non-singular at $\gamma\left(s_{0}\right)$. It also follows from Proposition 3.1 that $\gamma$ and $T_{M, \gamma\left(s_{0}\right)}^{D}$ have contact of order three at $\gamma\left(s_{0}\right)$ if and only if $\sigma_{D}\left(s_{0}\right)=0$ and $\sigma_{D}^{\prime}\left(s_{0}\right) \neq 0$.

Proposition 3.3 The following conditions are equivalent:

(1) $\boldsymbol{\gamma}$ and the timelikelike tangent plane $T P_{\boldsymbol{v}_{0}, \boldsymbol{\gamma}\left(s_{0}\right)}^{T}$ have contact order three,

(2) $\sigma_{D}\left(s_{0}\right)=0$ and $\sigma_{D}^{\prime}\left(s_{0}\right) \neq 0$,

(3) $\gamma$ and the the tangent D-slice $T_{M, \gamma\left(s_{0}\right)}^{D}$ have contact order three. 
As a consequence, we have the model curves on $M$ if pseudo-spherical evolutes are constant, that is, $\boldsymbol{h}_{\gamma}^{\prime} \equiv 0$ or $\boldsymbol{d}_{\gamma}^{\prime} \equiv 0$. In the next section, we investigate the singularities of pseudo-spherical evolute.

\section{Unfoldings of Functions}

In order to investigate the singularities of pseudo-spherical evolutes, we apply the theory of unfoldings of functions.

First, we give a quick review on the theory of unfoldings of functions of one variable. Detailed descriptions are found in the book[1]. Let $F:\left(\mathbb{R} \times \mathbb{R}^{r},\left(s_{0}, x_{0}\right)\right) \rightarrow \mathbb{R}$ be a function germ. We call $F$ an $r$-parameter unfolding of $f$, where $f(s)=F_{x_{0}}(s)$. We say that $f$ has type $A_{k}$ at $s_{0}$ if $f^{(p)}\left(s_{0}\right)=0$ for all $1 \leqq p \leqq k$, and $f^{(k+1)}\left(s_{0}\right) \neq 0$. We also say that $f$ has type $A_{\geq k}$ at $s_{0}$ if $f^{(p)}\left(s_{0}\right)=0$ for all $1 \leqq p \leqq k$. Let $F$ be an unfolding of $f$ and $f(s)$ has type $A_{k}(k \geqq 1)$ at $s_{0}$. We denote the $(k-1)$-jet of the partial derivative $\frac{\partial F}{\partial x_{i}}$ at $s_{0}$ by $j^{(k-1)}\left(\frac{\partial F}{\partial x_{i}}\left(s, x_{0}\right)\right)\left(s_{0}\right)=\sum_{j=1}^{k-1} \alpha_{j i} s^{j}$ for $i=1, \ldots, r$. Then $F$ is called an $\mathcal{R}^{+}$-versal unfolding if the $(k-1) \times r$ matrix of coefficients $\left(\alpha_{j i}\right)$ has rank $k-1(k-1 \leqq r)$. Under the same condition as the above, $F$ is called an $\mathcal{R}$-versal unfolding if the $k \times r$ matrix of coefficients $\left(\alpha_{0 i}, \alpha_{j i}\right)$ has rank $k(k \leqq r)$, where $\alpha_{0 i}=\frac{\partial F}{\partial x_{i}}\left(s_{0}, x_{0}\right)$.

We now introduce important sets concerning the unfoldings relative to the above notions. The bifurcation set $B_{F}$ of $F$ is

$$
B_{F}=\left\{x \in \mathbb{R}^{r} \mid \exists s ; \text { with } \frac{\partial F}{\partial s}(s, x)=\frac{\partial^{2} F}{\partial s^{2}}(s, x)=0\right\} .
$$

Then we have the following fundamental result of the unfolding theory (cf., [1]):

Theorem 4.1 Let $F:\left(\mathbb{R} \times \mathbb{R}^{r},\left(s_{0}, x_{0}\right)\right) \rightarrow \mathbb{R}$ be an $r$-parameter unfolding of $f$ which has the type $A_{k}$ at $s_{0}$. If $F$ is a $\mathcal{R}^{+}$-versal unfolding and $k=3$, then the germ of $B_{F}$ at $x_{0}$ is diffeomorphic to $\left(C \times \mathbb{R}^{r-2}, \mathbf{0}\right)$ as set germs. Here, $C=\left\{\left(x_{1}, x_{2}\right) \mid x_{1}{ }^{2}=x_{2}{ }^{3}\right\}$ is the ordinary cusp.

Here we have the following proposition:

Proposition 4.2 Let $\gamma: I \longrightarrow M$ be a unit speed curve with $\kappa_{n}\left(s_{0}\right) \neq 0$.

(1) If $h_{\boldsymbol{v}_{0}}^{T}(s)$ has type $A_{3}$ at $s_{0}$, then $H^{T}$ is an $\mathcal{R}^{+}$-versal unfolding of $h_{\boldsymbol{v}_{0}}^{T}(s)$.

(2) If $h_{\boldsymbol{v}_{0}}^{S}(s)$ has type $A_{3}$ at $s_{0}$, then $H^{S}$ is an $\mathcal{R}^{+}$-versal unfolding of $h_{\boldsymbol{v}_{0}}^{S}(s)$.

Proof. We denote $\gamma(s)=\left(x_{0}(s), x_{1}(s), x_{2}(s)\right), \boldsymbol{v}=\left(v_{0}, v_{1}, \sqrt{-1+v_{0}^{2}-v_{1}^{2}}\right) \in H^{2}(-1)$. Therefore we have

$$
H^{T}(s, \boldsymbol{v})=-x_{0}(s) v_{0}+x_{1} v_{1}+\sqrt{-1+v_{0}^{2}-v_{1}^{2}}
$$

and

$$
\begin{gathered}
\frac{\partial H^{T}}{\partial v_{0}}=-x_{0}(s)+\frac{v_{0}}{\sqrt{-1+v_{0}^{2}-v_{1}^{2}}} x_{2}(s), \quad \frac{\partial H^{T}}{\partial v_{1}}=x_{1}(s)-\frac{v_{1}}{\sqrt{-1+v_{0}^{2}-v_{1}^{2}}} x_{2}(s) \\
\frac{\partial^{2} H^{T}}{\partial s \partial v_{0}}=-x_{0}^{\prime}(s)+\frac{v_{0}}{\sqrt{-1+v_{0}^{2}-v_{1}^{2}}} x_{2}^{\prime}(s), \quad \frac{\partial^{2} H^{T}}{\partial s \partial v_{1}}=x_{1}^{\prime}(s)-\frac{v_{1}}{\sqrt{-1+v_{0}^{2}-v_{1}^{2}}} x_{2}^{\prime}(s)
\end{gathered}
$$




$$
\frac{\partial^{3} H^{T}}{\partial s^{2} \partial v_{0}}=-x_{0}^{\prime \prime}(s)+\frac{v_{0}}{\sqrt{-1+v_{0}^{2}-v_{1}^{2}}} x_{2}^{\prime \prime}(s) \quad, \quad \frac{\partial^{3} H^{T}}{\partial s^{2} \partial v_{1}}=x_{1}^{\prime \prime}(s)-\frac{v_{1}}{\sqrt{-1+v_{0}^{2}-v_{1}^{2}}} x_{2}^{\prime \prime}(s)
$$

(1) By Proposition 3.1, $h_{\boldsymbol{v}}^{T}\left(s_{0}\right)$ has type $A_{3}$ at $s_{0}$ if and only if

$$
\boldsymbol{v}= \pm \frac{1}{\sqrt{\kappa_{g}^{2}(s)-\kappa_{n}^{2}(s)}}\left(\kappa_{g}(s) \boldsymbol{n}_{\boldsymbol{\gamma}}(s)+\kappa_{n}(s) \boldsymbol{b}(s)\right),
$$

$\kappa_{g}^{2}(s)>\kappa_{n}^{2}(s), \sigma_{H}=0$, and $\sigma_{H}^{\prime} \neq 0$.

For the purpose, we require the

$$
A=\left(\begin{array}{ll}
-x_{0}^{\prime}(s)+\frac{v_{0}}{\sqrt{-1+v_{0}^{2}-v_{1}^{2}}} x_{2}^{\prime}(s) & x_{1}^{\prime}(s)-\frac{v_{1}}{\sqrt{-1+v_{0}^{2}-v_{1}^{2}}} x_{2}^{\prime}(s) \\
-x_{0}^{\prime \prime}(s)+\frac{v_{0}}{\sqrt{-1+v_{0}^{2}-v_{1}^{2}}} x_{2}^{\prime \prime}(s) & x_{1}^{\prime \prime}(s)-\frac{v_{1}}{\sqrt{-1+v_{0}^{2}-v_{1}^{2}}} x_{2}^{\prime \prime}(s)
\end{array}\right)
$$

to be non-singular. Therefore we calculate determinant of this matrix.

$$
\begin{aligned}
\operatorname{det} A & =\left(-\left(x_{1}^{\prime} x_{2}^{\prime \prime}-x_{2}^{\prime} x_{1}^{\prime \prime}\right), x_{2}^{\prime} x_{0}^{\prime \prime}-x_{0}^{\prime} x_{2}^{\prime \prime}, x_{0}^{\prime} x_{1}^{\prime \prime}-x_{1}^{\prime} x_{0}^{\prime}\right)\left(\begin{array}{c}
\frac{v_{0}}{\sqrt{-1+v_{0}^{2}-v_{1}^{2}}} \\
-\frac{v_{1}}{\sqrt{-1+v_{0}^{2}-v_{1}^{2}}} \\
-1
\end{array}\right) \\
& =\left(\left(x_{0}^{\prime}, x_{1}^{\prime}, x_{2}^{\prime}\right) \wedge\left(x_{0}^{\prime \prime}, x_{1}^{\prime \prime}, x_{2}^{\prime \prime}\right)\right)\left(\begin{array}{c}
\frac{v_{0}}{\sqrt{-1+v_{0}^{2}-v_{1}^{2}}} \\
-\frac{v_{1}}{\sqrt{-1+v_{0}^{2}-v_{1}^{2}}}
\end{array}\right) \\
& =-\frac{1}{\sqrt{-1+v_{0}^{2}-v_{1}^{2}}}\left(\boldsymbol{t} \wedge\left(\kappa_{n} \boldsymbol{n}_{\boldsymbol{\gamma}}+\kappa_{g} \boldsymbol{b}\right)\right)\left(\begin{array}{c}
-v_{0} \\
v_{1} \\
\sqrt{-1+v_{0}^{2}-v_{1}^{2}}
\end{array}\right) \\
& =-\frac{1}{\sqrt{-1+v_{0}^{2}-v_{1}^{2}}}\left\langle\kappa_{n} \boldsymbol{b}+\kappa_{g} \boldsymbol{n}_{\boldsymbol{\gamma}}, \pm \frac{1}{\sqrt{\kappa_{g}^{2}(s)-\kappa_{n}^{2}(s)}}\left(\kappa_{g} \boldsymbol{n}_{\gamma}(s)+\kappa_{n}(s) \boldsymbol{b}(s)\right)\right\rangle \\
& =\mp \frac{\sqrt{\kappa_{g}^{2}(s)-\kappa_{n}^{2}(s)}}{\sqrt{-1+v_{0}^{2}-v_{1}^{2}}} \neq 0
\end{aligned}
$$

By the same way, if we consider the spacelike height function $H^{S}$, we can prove (2).

As a consequence, we have the following theorem:

Theorem 4.3 Let $\gamma: I \longrightarrow M$ be a regular curve. Then we have the following assertions:

(A1) The hyperbolic evolute at $\boldsymbol{h}_{\boldsymbol{\gamma}}\left(s_{0}\right)$ is regular if $\sigma_{H}\left(s_{0}\right) \neq 0$.

(A2) The following conditions are equivalent:

(i) The germ of the hyperbolic evolute at $\boldsymbol{h}_{\boldsymbol{\gamma}}\left(s_{0}\right)$ is diffeomorphic to the ordinary cusp,

(ii) $\sigma_{H}\left(s_{0}\right)=0$ and $\sigma_{H}^{\prime}\left(s_{0}\right) \neq 0$,

(iii) $\boldsymbol{\gamma}$ and the spacelike tangent plane $T P_{\boldsymbol{v}_{0}, \boldsymbol{\gamma}\left(s_{0}\right)}^{S}$ have contact order three where $\boldsymbol{v}_{0}=\boldsymbol{h}_{\boldsymbol{\gamma}}\left(s_{0}\right)$,

(iv) If $\kappa_{n}\left(s_{0}\right) \neq 0$, then the tangent $H$-slice $T_{M, \gamma\left(s_{0}\right)}^{H}$ of $\boldsymbol{\gamma}$ at $\gamma\left(s_{0}\right)$ is non-singular, and $\boldsymbol{\gamma}$ and the the tangent $H$-slice $T_{M, \gamma\left(s_{0}\right)}^{H}$ have contact order three. 
(B1) The de Sitter evolute at $\boldsymbol{d}_{\boldsymbol{\gamma}}\left(s_{0}\right)$ is regular if $\sigma_{D}\left(s_{0}\right) \neq 0$.

(B2) The following conditions are equivalent:

(i) The germ of the de Sitter evolute at $\boldsymbol{d}_{\boldsymbol{\gamma}}\left(s_{0}\right)$ is diffeomorphic to the ordinary cusp,

(ii) $\gamma$ and the timelikelike tangent plane $T P_{\boldsymbol{v}_{0}, \gamma\left(s_{0}\right)}^{T}$ have contact order three,

(iii) $\sigma_{D}\left(s_{0}\right)=0$ and $\sigma_{D}^{\prime}\left(s_{0}\right) \neq 0$.

(iv) $\boldsymbol{\gamma}$ and the the tangent $D$-slice $T_{M, \gamma\left(s_{0}\right)}^{D}$ have contact order three.

Proof. (A1) By the assertion of Proposition 3.1, we have $\boldsymbol{h}_{\boldsymbol{\gamma}}^{\prime}(s)=0$ if $\sigma_{H}(s)=0$. It means that the hyperbolic evolute at $\boldsymbol{h}_{\boldsymbol{\gamma}}\left(s_{0}\right)$ is regular if $\sigma_{H}\left(s_{0}\right) \neq 0$.

(A2) By Proposition 3.1, the bifurcation set of $H^{T}$ is

$$
B_{F}=\left\{\boldsymbol{h}_{\boldsymbol{\gamma}}(s)=\frac{\kappa_{g}(s)}{\sqrt{\kappa_{g}^{2}(s)-\kappa_{n}^{2}(s)}} \boldsymbol{n}_{\boldsymbol{\gamma}}(s)+\frac{\kappa_{n}(s)}{\sqrt{\kappa_{g}^{2}(s)-\kappa_{n}^{2}(s)}} \boldsymbol{b}(s) \mid \kappa_{g}^{2}>\kappa_{n}^{2}\right\} .
$$

By Theorem 4.1 and Proposition 4.2, the germ of the bifurcation set is diffeomorphic to the ordinary cusp if $\sigma_{H}=0$ and $\sigma_{H}^{\prime} \neq 0$. Moreover we have other equivalences by Proposition 3.2. This complete the proof for (A1) and (A2).

If we consider the spacelike height function $H^{S}$, we can prove the remaining assertions of the theorem.

\section{Examples}

In this section we consider some examples which explain the difference from the Euclidean plane curves.

\subsection{Curves on a spacelike plane}

Suppose that $M=\mathbb{R}_{0}^{2}=\left\{\boldsymbol{x}=\left(x_{0}, x_{1}, x_{2}\right) \in \mathbb{R}_{1}^{3} \mid x_{0}=0\right\}$. We consider a plane curve $\boldsymbol{\gamma}: I \longrightarrow \mathbb{R}_{0}^{2}$. In this case we have $\boldsymbol{n}_{\boldsymbol{\gamma}}=\boldsymbol{e}_{0}, \boldsymbol{t}(s)=\boldsymbol{\gamma}^{\prime}(s)$ and $\boldsymbol{b}(s)=\boldsymbol{t}(s) \wedge \boldsymbol{e}_{0}$. It follows that $\kappa_{n}(s) \equiv \tau_{g}(s) \equiv 0$ and $\kappa_{g}(s)=\left\langle\boldsymbol{t}^{\prime}(s), \boldsymbol{b}(s)\right\rangle=\kappa(s)$. Then we have the following classical Frenet-Serret formulae on Euclidean plane:

$$
\left\{\begin{array}{l}
\boldsymbol{t}^{\prime}(s)=\kappa(s) \boldsymbol{b}(s) \\
\boldsymbol{b}^{\prime}(s)=-\kappa(s) \boldsymbol{t}(s) .
\end{array}\right.
$$

Here we have $\sigma_{H} \equiv 0$ and the constant hyperbolic evolute $\boldsymbol{h}_{\boldsymbol{\gamma}}(s)=\boldsymbol{n}_{\boldsymbol{\gamma}}=\boldsymbol{e}_{0}$ of $\boldsymbol{\gamma}$ relative to $M$ It means that $H$-slice is $M=P(\boldsymbol{v}, c) \cap M$. Moreover, we do not have de Sitter evolutes. It is known [1, page 37, 2.30] that the evolute of $\boldsymbol{\gamma}$ is defined by

$$
\boldsymbol{e}_{\boldsymbol{\gamma}}(s)=\gamma(s)+\frac{1}{\kappa(s)} \boldsymbol{b}(s)
$$

Therefore, we have a completely different situation from those of the Euclidean plane curves. 


\subsection{Curves on the hyperbolic plane $H_{+}^{2}(-1)$}

Suppose that $M=H_{+}^{2}(-1)$. In this case, we have $\boldsymbol{n}_{\boldsymbol{\gamma}}(s)=\boldsymbol{\gamma}(s), \boldsymbol{t}(s)=\boldsymbol{\gamma}^{\prime}(s)$ with $\|\boldsymbol{t}(s)\|=1$ and $\boldsymbol{b}(s)=\boldsymbol{t}(s) \wedge \boldsymbol{n}_{\boldsymbol{\gamma}}(s)$. We call $\{\boldsymbol{t}, \boldsymbol{\gamma}, \boldsymbol{b}\}$ the Lorentzian Saban frame. Therefore, we have $\kappa_{n}(s) \equiv 1, \tau_{g}(s) \equiv 0$. By the Frenet-Serret type formulae in $\S 3$, we have the following[2]:

$$
\left\{\begin{array}{l}
\boldsymbol{t}^{\prime}(s)=\gamma+\kappa_{g}(s) \boldsymbol{b}(s) \\
\boldsymbol{\gamma}^{\prime}(s)=\boldsymbol{t}(s) \\
\boldsymbol{b}^{\prime}(s)=-\kappa_{g}(s) \boldsymbol{t}(s)
\end{array}\right.
$$

Here, we have $\sigma_{H}=\left(\frac{1}{\kappa_{g}}\right)^{\prime}$, and $\sigma_{D}=\kappa_{g}{ }^{\prime}$. Moreover we have the hyperbolic evolute of $\gamma$ relative to $M, \boldsymbol{h}_{\boldsymbol{\gamma}}(s)=\left(\frac{\kappa_{g}}{\sqrt{\kappa_{g}^{2}-1}} \boldsymbol{n}_{\boldsymbol{\gamma}}+\frac{1}{\sqrt{\kappa_{g}^{2}-1}} \boldsymbol{b}\right)(s)$ for $\kappa_{g}^{2}(s)>1$ and the de Sitter evolute of $\boldsymbol{\gamma}$ relative to $M, \boldsymbol{d}_{\boldsymbol{\gamma}}(s)=\left(\frac{\kappa_{g}}{\sqrt{1-\kappa_{g}^{2}}} \boldsymbol{n}_{\boldsymbol{\gamma}}-\frac{1}{\sqrt{1-\kappa_{g}^{2}}} \boldsymbol{b}\right)(s)$ for $\kappa_{g}^{2}(s)<1$. The singularities of the hyperbolic evolute $\boldsymbol{h}_{\boldsymbol{\gamma}}(s)$ was investigated in [2]. By Theorem 4.3, the evolute at $\boldsymbol{h}_{\boldsymbol{\gamma}}\left(s_{0}\right)$ is the regular if $\sigma_{H}=\left(\frac{1}{\kappa_{g}}\right)^{\prime} \neq 0$, and is the ordinary cusp locally if $\sigma_{H}=\left(\frac{1}{\kappa_{g}}\right)^{\prime}=0$, and $\sigma_{H}{ }^{\prime}=\left(\frac{1}{\kappa_{g}}\right)^{\prime \prime} \neq 0$. Since $\sigma_{H}=\frac{-\kappa_{g}^{\prime}}{\kappa_{g}^{2}}$ and $\sigma_{H}^{\prime}=\frac{-\kappa_{g}^{\prime \prime} \kappa_{g}^{2}-2\left(\kappa_{g}^{\prime}\right)^{2}}{\kappa_{g}^{3}}, \sigma_{H}(s)=\sigma_{H}^{\prime}(s)=0$ if and only if $\kappa_{g}^{\prime}(s)=\kappa_{g}^{\prime \prime}(s)=0$. Moreover the de Sitter evolute at $\boldsymbol{d}_{\boldsymbol{\gamma}}\left(s_{0}\right)$ is the regular if $\sigma_{D}=\kappa_{g}{ }^{\prime} \neq 0$, and is the ordinary cusp locally if $\sigma_{D}=\kappa_{g}{ }^{\prime}=0$, and ${\sigma_{D}}^{\prime}=\kappa_{g}{ }^{\prime \prime} \neq 0$. Therefore the cusp of the pseudo-spherical evolute corresponding to the point $\gamma(s)$ with $\kappa_{g}^{\prime}(s)=0$ and $\kappa_{g}^{\prime \prime}(s) \neq 0$. We call it the ordinary vertex of $\gamma$.

Here we consider $H$-slices under the condition $\kappa_{g}^{2}>1$ and consider $D$-slices under the condition $\kappa_{g}^{2}<1$. Since we have a well-known classification theorem of totally umbilical surfaces (cf.,[4]), we can conclude that an $H$-slice is a (hyperbolic) circle if $\kappa_{g} \neq 0$ and $\kappa_{g}^{2}>1$, and a $D$-slice is an equidistant curve if $\kappa_{g} \neq 0$ and $\kappa_{g}^{2}<1$.

\subsection{Curves on a surface of graphical representation}

Suppose that $\boldsymbol{X}(x, y)=(f(x, y), x, y)$ with $f(0,0)=0$ and $\partial f / \partial x(0,0)=\partial f / \partial y(0,0)=0$, which is called the Monge form. Here we denote $f_{x}=\partial f / \partial x, f_{y}=\partial f / \partial y, \boldsymbol{X}_{x}=\partial \boldsymbol{X} / \partial x$ and $\boldsymbol{X}_{y}=\partial \boldsymbol{X} / \partial y$. Since $\boldsymbol{X}$ is a spacelike embedding, we have $\left\|\boldsymbol{X}_{x}\right\|=-f_{x}^{2}+1>0,\left\|\boldsymbol{X}_{y}\right\|=-f_{y}^{2}+$ $1>0$, and a unit timelike normal vector field $\boldsymbol{n}(p)=\frac{\boldsymbol{X}_{x}(u) \wedge \boldsymbol{X}_{y}(u)}{\left\|\boldsymbol{X}_{x}(u) \wedge \boldsymbol{X}_{y}(u)\right\|}=-\frac{1}{\sqrt{1-f_{x}^{2}-f_{y}^{2}}}\left(1, f_{x}, f_{y}\right)$ with $-1+f_{x}^{2}+f_{y}^{2}<0$ where $p=\boldsymbol{X}(u)=\boldsymbol{X}(x, y)$.

\section{(1) De Sitter evolutes}

We now consider the curve $\gamma(x)=\boldsymbol{X}(x, 0)=(f(x, 0), x, 0)$, where $f(x, y)$ is a smooth function. Here we denote $\frac{d \gamma}{d x}=\dot{\gamma}, f_{x}=f_{x}(x, 0)$, and $f_{y}(x, 0)$. Since $\dot{\gamma}(x)=\left(f_{x}, 1,0\right)$, we have the unit tangent vector field $\boldsymbol{t}(x)=\frac{1}{\sqrt{1-f_{x}^{2}}}\left(f_{x}, 1,0\right)$, and the two unit normal vector fields $\boldsymbol{n}_{\boldsymbol{\gamma}}(x)=\frac{-1}{\sqrt{1-f_{x}^{2}-f_{y}^{2}}}\left(1, f_{x}, f_{y}\right), \boldsymbol{b}(x)=\frac{1}{\sqrt{1-f_{x}^{2}-f_{y}^{2}} \sqrt{1-f_{x}^{2}}}\left(f_{y}, f_{x} f_{y}, 1-f_{x}^{2}\right)$. By straightforward calculations, we have $\kappa_{g}(x)=\left\langle\frac{d \boldsymbol{t}}{d s}(x), \boldsymbol{b}(x)\right\rangle=\frac{-f_{y} f_{x x}}{\left.\left(1-f_{x}^{2}\right)\right)^{\frac{3}{2}}\left(1-f_{x}^{2}-f_{y}^{2}\right)^{\frac{1}{2}}}$, and $\kappa_{n}(x)=\left\langle\frac{d \boldsymbol{t}}{d s}(x), \boldsymbol{n}_{\boldsymbol{\gamma}}(x)\right\rangle=$ $\frac{f_{x x}}{\left(1-f_{x}^{2}\right)\left(1-f_{x}^{2}-f_{y}^{2}\right)^{\frac{1}{2}}}$, where $s$ is the arc-length. Therefore we have $\frac{\kappa_{g}}{\kappa_{n}}(x)=\frac{-f_{y}}{\left(1-f_{x}^{2}\right)^{\frac{1}{2}}}$, that is, $\left(\frac{\kappa_{g}}{\kappa_{n}}\right)(x)(1-$ $\left.f_{x}^{2}\right)^{\frac{1}{2}}=-f_{y}$. By differentiation of the both sides, we have $\left(\frac{\kappa_{g}}{\kappa_{n}}\right)^{\prime}\left(1-f_{x}^{2}\right)^{\frac{1}{2}}+\left(\frac{\kappa_{g}}{\kappa_{n}}\right) \frac{-f_{x} f_{x x}}{\left(1-f_{x}^{2}\right)^{\frac{1}{2}}}=-f_{y x}$. 
Moreover, we have $\left(\frac{\kappa_{g}}{\kappa_{n}}\right)^{\prime \prime}\left(1-f_{x}^{2}\right)^{\frac{1}{2}}-2\left(\frac{\kappa_{g}}{\kappa_{n}}\right)^{\prime} \frac{f_{x} f_{x x}}{\left(1-f_{x}^{2}\right)^{\frac{1}{2}}}+\left(\frac{\kappa_{g}}{\kappa_{n}}\right)\left\{\frac{-f_{x} f_{x x}}{\left(1-f_{x}^{2}\right)^{\frac{1}{2}}}\right\}^{\prime}=-f_{y x x}$. Therefore we have

$$
\frac{\kappa_{g}}{\kappa_{n}}(0)=-f_{y}(0,0)=0,\left(\frac{\kappa_{g}}{\kappa_{n}}\right)^{\prime}(0)=-f_{y x}(0,0),\left(\frac{\kappa_{g}}{\kappa_{n}}\right)^{\prime \prime}(0)=-f_{y x x}(0,0) .
$$

On the other hand, we have

$$
\frac{d \boldsymbol{n}_{\boldsymbol{\gamma}}}{d s}(x)=\frac{1}{\left(1-f_{x}^{2}\right)^{\frac{1}{2}}\left(1-f_{x}^{2}-f_{y}^{2}\right)^{\frac{3}{2}}}\left(\left|\begin{array}{cc}
-f_{y} & f_{x x} \\
f_{x} & f_{y x}
\end{array}\right|,\left|\begin{array}{cc}
-f_{x} f_{y} & f_{x x} \\
1-f_{y}^{2} & f_{y x}
\end{array}\right|,\left|\begin{array}{cc}
f_{x}^{2}-1 & f_{x x} \\
-f_{x} f_{y} & f_{y x}
\end{array}\right|\right) .
$$

Then we have

$$
\begin{aligned}
\tau_{g}(x)= & \left\langle\boldsymbol{b}(x), \frac{d \boldsymbol{n}_{\boldsymbol{\gamma}}}{d s}(x)\right\rangle \\
= & \frac{1}{\left(1-f_{x}^{2}\right)\left(1-f_{x}^{2}-f_{y}^{2}\right)^{2}}\left\{f_{y}^{2} f_{y x}-f_{x}^{2} f_{y}^{2} f_{y x}+f_{x} f_{y} f_{x x}\right. \\
& \left.+f_{x x} f_{x} f_{y}^{3}-f_{x}^{3} f_{y} f_{x x}-f_{y x}+2 f_{y x} f_{x}^{2}-f_{y x} f_{x}^{4}\right\} .
\end{aligned}
$$

By the same calculations as those for $\frac{\kappa_{g}}{\kappa_{n}}$, we have

$$
\tau_{g}(0)=-f_{y x}(0,0) \text {, and } \tau_{g}^{\prime}(0)=-f_{y x x}(0,0) .
$$

We now consider the special case

$$
f(x, y)=a_{20} x^{2}+a_{11} x y+a_{02} y^{2}+a_{30} x^{3}+a_{21} x^{2} y+a_{12} x y^{2}+a_{03} y^{3} .
$$

Then we have $f_{y x}(0,0)=a_{11}, f_{y x x}(0,0)=a_{21}, f_{x x}(0,0)=a_{20}, f_{x x x}(0,0)=a_{30}$. Since $\kappa_{g}(0)=0$, we can define $\boldsymbol{d}_{\boldsymbol{\gamma}}$ where $\kappa_{n}(0)=a_{20} \neq 0$. Moreover we have

$$
\sigma_{D}(0)=-2 a_{11}, \sigma_{D}^{\prime}(0)=-2 a_{21}
$$

It means that we have $\sigma_{D}(0)=0, \sigma_{D}^{\prime}(0) \neq 0$ if $a_{11}=0$ and $a_{21} \neq 0$. Therefore $f(x, y)$, which is $a_{20} \neq 0, a_{11}=0$ and $a_{21} \neq 0$, is an example of de Sitter evolute which has a cusp at the origin. For instance, we have $f(x, y)=x^{2} y-\frac{1}{2} x^{2}$.

\section{(2) Hyperbolic evolutes}

We now consider $\boldsymbol{X}(x, y)=(f(x, y), x, y)$ where $f(x, y)=\frac{1}{2}(y-g(x))$ and $g(x)$ is a smooth function, and we investigate the curve $\boldsymbol{\gamma}(x)=\boldsymbol{X}(x, g(x))=(0, x, g(x))$. Here we denote $\frac{d \boldsymbol{\gamma}}{d x}=\dot{\boldsymbol{\gamma}}$, $\frac{d g}{d x}=\dot{g}, f_{x}=f_{x}(x, 0)$, and $f_{y}(x, 0)$. Since $\dot{\gamma}(x)=(0,1, \dot{g}(x))$, we have the unit tangent vector field $\boldsymbol{t}(x)=\frac{1}{\sqrt{1+\dot{g}^{2}(x)}}(0,1, \dot{g}(x))$, and the two normal vector fields

$$
\begin{gathered}
\boldsymbol{n}_{\boldsymbol{\gamma}}(x)=\frac{-1}{\sqrt{1-f_{x}^{2}(x, 0)-f_{y}^{2}(x, 0)}}\left(1, f_{x}(x, 0), f_{y}(x, 0)\right), \\
\boldsymbol{b}(x)=\frac{1}{\sqrt{1-f_{x}^{2}(x, 0)-f_{y}^{2}(x, 0)} \sqrt{1+\dot{g}^{2}(x)}}\left(\dot{g}(x) f_{x}(x, 0)-f_{y}(x, 0),-\dot{g}(x), 1\right) .
\end{gathered}
$$

By the same arguments as those in the case for de Sitter evolutes, we have

$$
\frac{\kappa_{g}}{\kappa_{n}}(x)=\frac{-\left(1+\dot{g}^{2}(x)\right)^{\frac{1}{2}}}{-\dot{g}(x) f_{x}(x, 0)+f_{y}(x, 0)}, \quad \tau_{g}(x)=\frac{-1}{\left(1+\dot{g}^{2}(x)\right)\left(1-f_{x}^{2}-f_{y}^{2}\right)^{2}}\left(-\dot{g}(x) f_{x x}(x, 0)+f_{y x}(x, 0)\right)
$$


Moreover we have

$$
\frac{\kappa_{g}}{\kappa_{n}}(0)=-2,\left(\frac{\kappa_{g}}{\kappa_{n}}\right)^{\prime}(0)=0,\left(\frac{\kappa_{g}}{\kappa_{n}}\right)^{\prime \prime}(0)=8 \ddot{g}(0), \tau_{g}(0)=0, \tau_{g}^{\prime}(0)=-\frac{8}{3} \ddot{g}^{2}(0) .
$$

We now consider the special case $g(x)=a_{2} x^{2}+a_{3} x^{3}$. Then we have $\ddot{g}_{x x}(0)=a_{2}, \dddot{g}(0)=a_{3}$. Since $\kappa_{n}(0)=-\frac{4}{3} a_{2}$, we can define $\boldsymbol{h}_{\boldsymbol{\gamma}}$ where $a_{2} \neq 0$. Moreover we have

$$
\sigma_{H}(0)=0, \sigma_{H}^{\prime}(0) \neq 0
$$

if $a_{20} \neq 0$. Therefore $g(x)$, which is $a_{2} \neq 0$, is an example of hyperbolic evolute which has a cusp at the origin.

\section{References}

[1] J. W. Bruce and P. J. Giblin, Curves and singularities (second edition). Cambridge University press (1992)

[2] S. Izumiya, D.-H, Pei, T. Sano and E. Torii, Evoutes of Hyperbolic Plane Curves. Acta Math. Sinica, English Series, 20, No. 3 (2004) 543-550

[3] S. Izumiya, M. C. Romero Fuster, the lightlike flat geometry in spacelike submanifolds of codimension two in Minkowski space. Sel. math.,New ser., 13 (2007),23-55

[4] S. Izumiya, D.-H, Pei and M. Takahashi, Singularities of evolutes of hypersurfaces in hyperbolic space. Proc. Edinb. math. Soc. (2), 47 (2004), 131-153.

[5] B. O'Neill, Semi-Riemannian Geometry, Academic Press, New York, 1983.

[6] T. Sato, Curves on a spacelike surface in three dimentional Lorentz-Minkowski space. Preprint (2012).

Takami Sato, Department of Mathematics, Hokkaido University, Sapporo 060-0810,Japan e-mail:takami.s1218@gmail.com 